\title{
Research of properties of raw cotton -as the object of drying
}

\author{
Khussanov A.E ${ }^{1}$. , Atamanyuk V.M ${ }^{2}$., Janabayev D.J ${ }^{1}$., Kaldybaeva B.M ${ }^{1}$ \\ 1. Scientific Laboratory "Water Problems", M.Auezov South Kazakhstan State University, KAZAKHSTAN, \\ Shymkent, Taukekhan avenue, 5, E-mail: khusanov_1975@inbox.ru \\ 2. Department of Chemical Endineering, Lviv Polytechnic National University, UKRAINE, Lviv,12 S.Bandera \\ street, E-mail: atamanyuk@ukr.net.
}

This article presents the results of the study of the properties of raw cotton with a multi-purpose scanning electron microscope, which combines the possibilities of working in both standard and lowvacuum modes. On cross sections of the fibers, three zones are noticeable, differing in microstructure and relation to the action of the reagents. The hygroscopicity, tensile strength, heat resistance, chemical properties, resistance to biological destruction, the structure of cotton are determined. On cross sections of the fibers, three zones are noticeable, differing in microstructure and relation to the action of the reagents. Zones $A$ correspond to places with a higher density of the fibrillar structure and, therefore, the lowest availability for reagents. Zone $B$ has a well-oriented structure of concentric layers, but more changes under the influence of reagents. Zone $C$ is characterized by an unorganized and looser structure, so the fiber here is easier to react with. Inside the fiber passes a channel that is filled with air in a mature fiber. For cotton fiber, 11 maturity groups have been established, characterized by a coefficient of maturity - from 0 to 5 points.

Keywords: filtration drying, hydrodynamics, fibrous materials, moisture, thermal agent, heat-mass transfer, diffusion

\section{Introduction}

During the drying of raw cotton, its temperature rises slightly, which will lead to a deterioration of the technological properties of the fiber. If the temperature rises during drying of raw cotton, a lot of moisture is lost, which leads to the rupture of each fiber. Each variety of raw cotton has its own specific to physical factors. The fiber has a mechanically bound moisture that easily evaporates from the surface area as from the free surface of the liquid. The movement of moisture and the mechanism of evaporation of water from the seeds depends on the level of interacting factors. The choice of raw material drying mode strongly depends on the following parameters: color, fiber length, type of mechanical damage, etc. In case of improper drying, fiber breakage is observed, its length is reduced, as a result, the quality of the fiber in The processing of raw cotton for more efficient and high-quality fiber, their humidity should be within 8-9\% $[1,2]$.

\section{Result of research and discussion}

To determine the properties of raw cotton, a multi-purpose scanning electron microscope (useful magnification of 300 000) was used, which combines the capabilities of both standard and low-vacuum modes. Allows you to examine samples without spraying with a conductive layer. Additionally equipped with energy dispersive microanalysis system INCA Energy 350 and a prefix for the study of texture and structure of polycrystalline samples HKL Basic

The breaking strength of cotton fiber, depending on the degree of maturity and variety of its ranges from 0.5 to $10 \mathrm{SN} /$ fiber. The average strength of Mature fiber is 4-6 SN/fiber. Fibers having a strength of up to $2.5 \mathrm{SN} /$ fiber are non-discharge. The relative breaking load varies from 24 to $36 \mathrm{SN} / \mathrm{Tex}$ depending on the breeding varieties of cotton and the degree of maturity of the fiber[1,2]. 
The elongation of the fiber at break is on average $7-8 \%$, which is explained by the spiral arrangement of cellulose layers in the fiber wall. Cotton fibers have a small elastic and large plastic elongation. Because of the low elasticity fiber textile products of cotton crumple easily, they formed derepresses folds and samini.

Under normal conditions, the moisture content of cotton fiber is $7-9 \%$. To the action of water, both cold and hot, cotton fiber is resistant. When heated in hot water (up to $150{ }^{\circ} \mathrm{C}$ ) under pressure, the properties of cotton also do not deteriorate. In the water environment, cotton fiber swells, increases in diameter by $15-30 \%$ depending on the temperature and duration of treatment. The swelling of the fiber is accompanied by an increase in its tensile strength by $15-17 \%$, which is explained by an increase in the degree of orientation of cellulose macromolecules in the direction of the tensile load.

The thermal resistance of cotton fiber is quite high. Moistened fiber is not destroyed at a temperature even above $250{ }^{\circ} \mathrm{C}$. As long as the moisture from the fiber is removed by evaporation, its temperature does not exceed $100^{\circ} \mathrm{C}$. However, after moisture loss, the fiber is quickly destroyed to form a variety of liquid and gaseous products. At a temperature of 400-450 ${ }^{\circ} \mathrm{C}$, the fiber is charred. The fiber can withstand short-term heating to a temperature of $120-150$ ${ }^{\circ} \mathrm{C}$.

Under the action of mineral acids cotton fiber is destroyed by hydrolysis of cellulose. The alkali resistance of cotton fibers is very high: even when boiling in alkaline solutions without oxygen, the fibers are almost unchanged. But when boiling in solutions of caustic alkalis with air access, the mechanical strength of the fiber decreases due to the oxidation of cellulose.
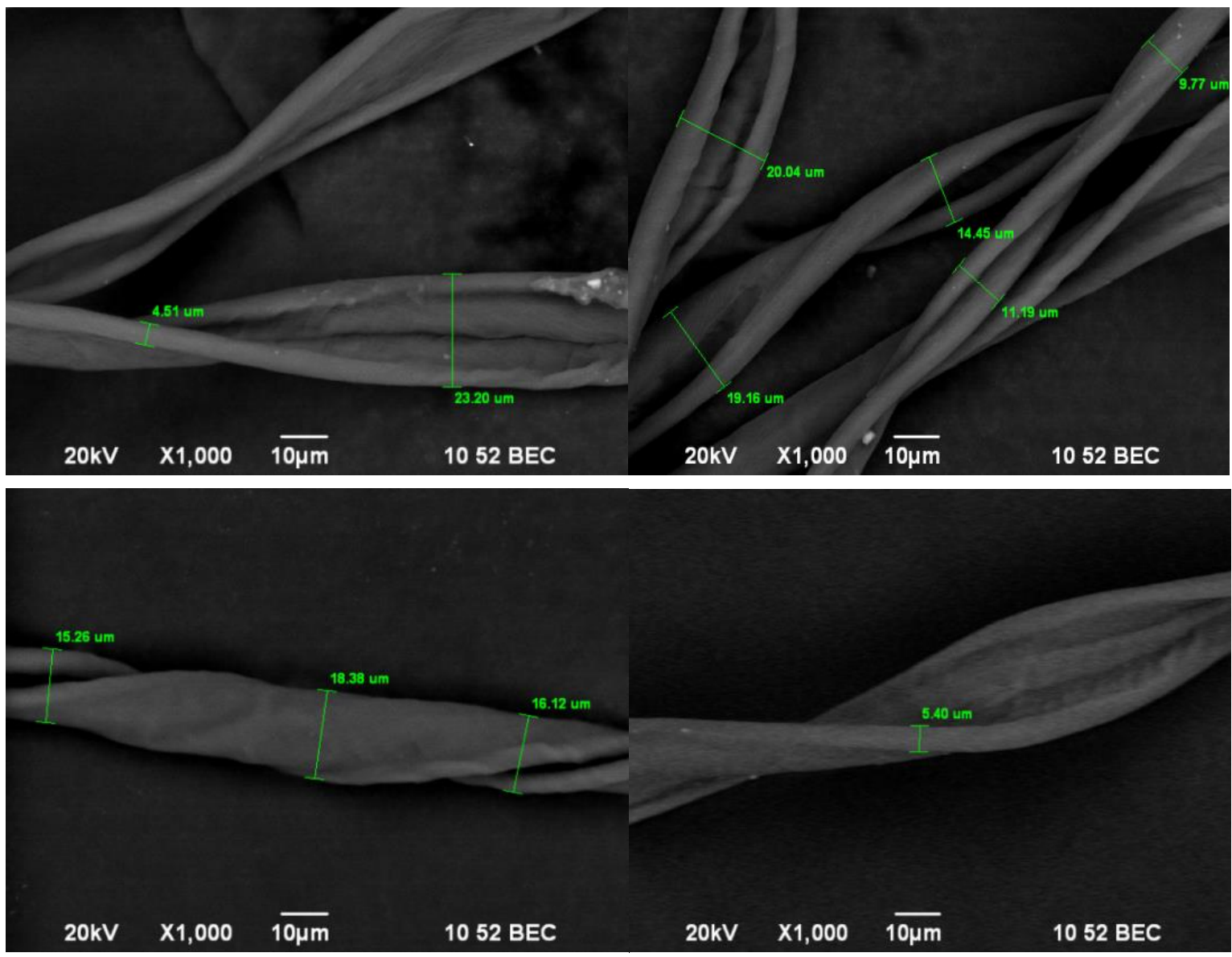


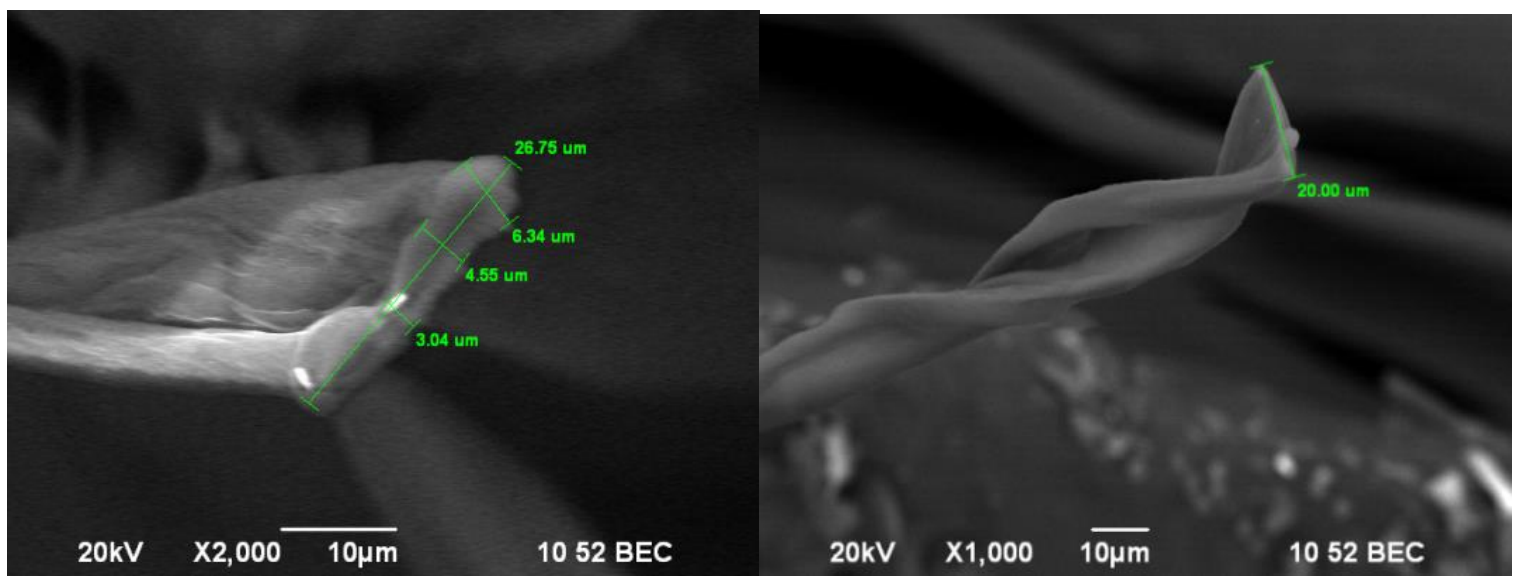

Fig.1. Results of investigation of raw cotton properties by multi-purpose scanning electron microscope

Resistance to biological destruction. Cotton fibers in chemical composition are a good enough substrate for the life of various microorganisms. Fiber with moisture content (more than 9\%) can be damaged or destroyed as a result of the life of microorganisms.

\section{Conclusion}

Mature cotton fiber is an elementary elongated plant cell and under the microscope has the form of a flattened tube with a corkscrew-like tortuosity, tapering at the ends (to the top - to a greater extent, to the bottom - to a lesser extent). The upper end of the fiber is cone-shaped, tightly closed. The lower end, adjacent to the seed, is torn off and has an open channel. The surface of the fiber is covered with a thin layer of fatty substances - the primary wall, or cuticle. This layer performs a protective role and has a sufficiently high chemical resistance, low wettability and water permeability, facilitates the sliding of fibers in the process of spinning. The primary wall has a small thickness (about 0.1 microns), and its upper layer is Packed less tightly than the inner one, due to the expansion of the fiber surface during its growth.

\section{References}

[1] G. R. Rakhmatov, "Installation of the IR dryer of raw cotton”, European Science Review, no. 5-6, pp. 185-186, 2016.

[2] A.E. Khussanov, D.J. Janabayev, M.B. Kenzhekhanova, A.K. Bukharbayeva," Results of the study of the properties of raw cotton by a multiple treatment electronic microscope" International Scentific-Practical cohference «Auezov Readings-17», Shymkent, 2019, pp. 196-200. 